\title{
Keratin 17 is ubiquitinated by Trim21 in human keratinocytes in
}

\section{psoriasis}

\author{
Luting Yang ${ }^{1}$, Liang Jin ${ }^{1}$, Xueli Fan ${ }^{1}$, Tongmei Zhang ${ }^{1}$, Gang Wang1*
}

1Department of Dermatology, Xijing Hospital, Fourth Military Medical University, Xi'an

710032, Shaanxi, China

"Corresponding author's email: xjwgang@fmmu.edu.cn

\section{INTRODUCTION}

Keratin 17 (K17) is a type I intermediate filament which is highly expressed in psoriatic epidermis. Studies on K17 uncover several role for K17, including mechanical support, regulation of cell proliferation and differentiation. We previously proposed a K17/Tcell/cytokine autoimmune loop in which $\mathrm{K} 17$ could be induced by inflammatory cytokine stimulation in psoriasis. Yet, little is known about the regulation of $\mathrm{K} 17$ expression via post-translational modification. Ubiquitination is one of the most important posttranslational modifications that modulate the functions of proteins. UbiNet software predicated several ubiquitination sites on human K17. We sought to determine whether K17 underwent ubiquitination by dysregulated E3 ligase in psoriasis, which regulated the protein levels of K17 and contributed to the pathogenesis of psoriasis

\section{METHODS}

To assess whether $\mathrm{K} 17$ is ubiquitinated in human keratinocytes, we employed immunoprecipitation. Combined with massspectrometry, we sought to determine the E3 ligase in interaction with K17. Furthermore, we used Real-Time PCR, western blotting and immunofluorescence to evaluate the effect of E3 ligase on the stability of K17.

\section{RESULTS}

1. Evaluation of K17 stability in normal and psoriatic keratinocytes
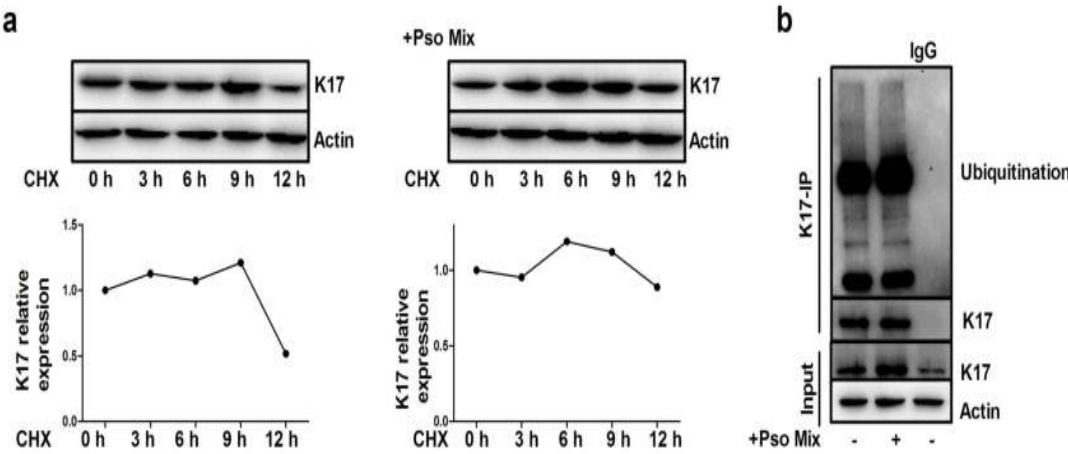

Fig.1 (a) Cells were treated with IL-17/IL-22/TNF- $\alpha$ (Pso Mix) for $24 \mathrm{~h}$ (right) before treating with $10 \mu \mathrm{M}$ cycloheximide (CHX). Whole-cell lysates were subjected to immunoblot (IB) analysis. Band intensities were quantified relative to $\beta$-Actin. (b) Immunoprecipation with anti-K17 antibody or IgG were performed in $\mathrm{HaCaT}$ cells and cells treated with Pso Mix.
2. Mass spectrometry and immunoprecipitation identified E3 ligase Trim21 to interact with $\mathrm{K} 17$

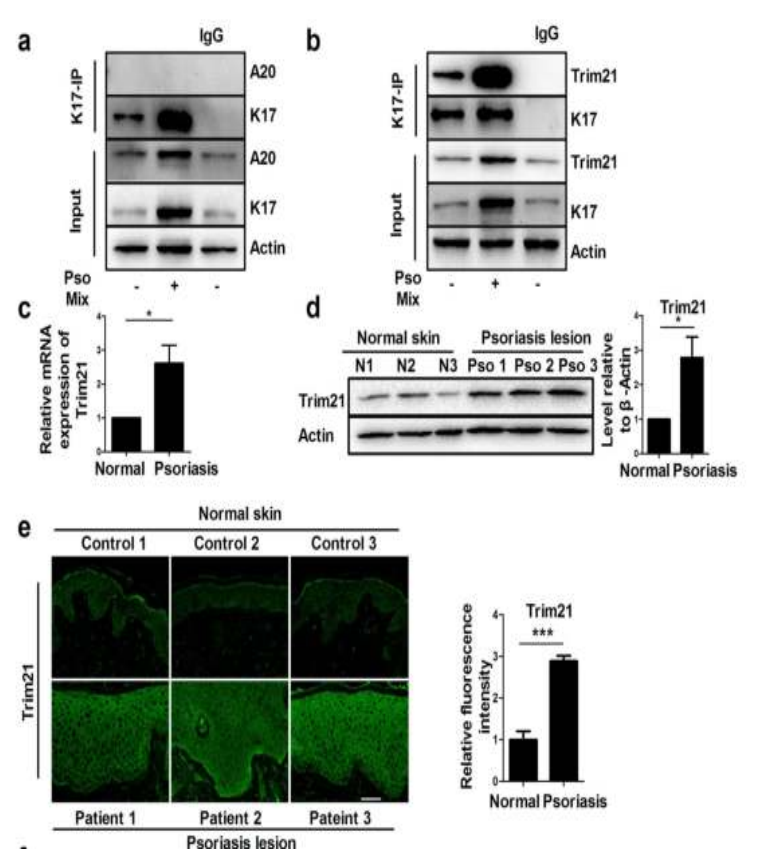

Fig.2 Upregulated E3 ligase Trim21 in psoriatic epidermis interacts with K17. (a and b) Psoriatic keratinocytes and normal keratinocytes $\mathrm{HaCaT}$ cells were subjected to immunoprecipitation with anti-K17 or IgG. IB were performed with A20 (a) and Trim21 (b) antibodies. (c) mRNA and (d) protein levels of Trim21 were measured in epidermis of skin biopsies from healthy controls and psoriatic patients. (e) Immunofluorescence staining and quantitation of Trim21 in three normal and psoriatic lesional skin.

3. Effect of Trim21 on K17 stability

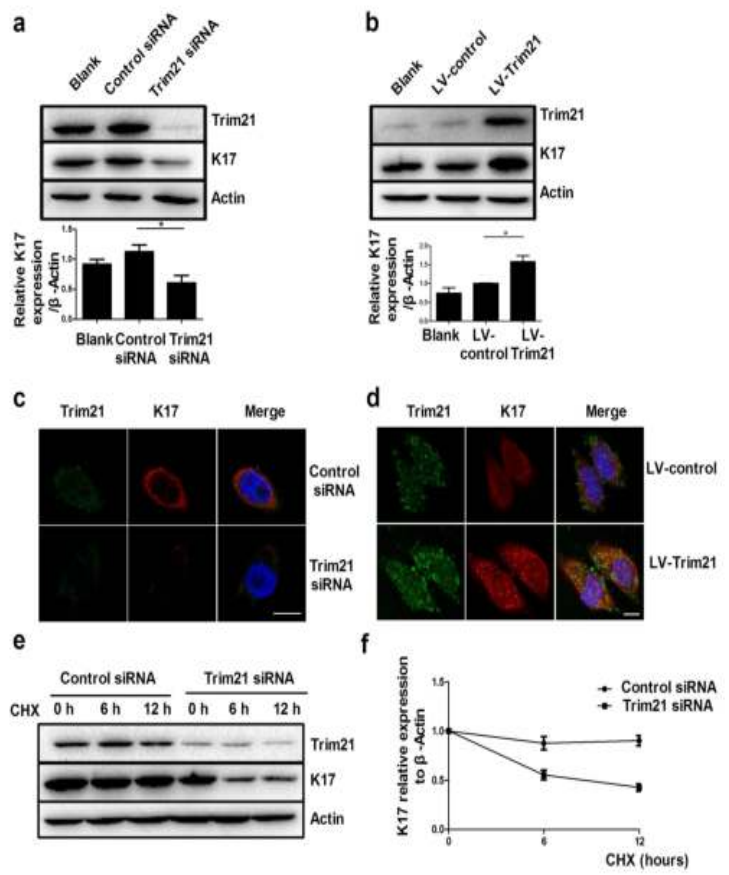

Fig.3 Trim21 promotes the stability of $\mathrm{K} 17$ in $\mathrm{HaCaT}$ cells. (a) IB analysis of K17 protein levels after knockdown of Trim21 in HaCaT cells (left) and cells treated with Pso Mix. (b) IB analysis of K17 expression in stable overexpression of Trim21 (LV-Trim21) and control $\mathrm{HaCaT}$ cells. (c and d) Immunofluorescence analysis of $\mathrm{K} 17$, Trim21 levels after knockdown (c) or overexpression of Trim21 (d). (e) Half-life of K17 was measured by CHX assay in control and Trim21 knockdown HaCaT cells. (f) Band intensities of K17 were measured relative to $\beta$-Actin. ${ }^{*} P<0.05 ; * * * P<0.001$
4. Trim21 ubiquitylates K17 via K63-linked ubiquitination

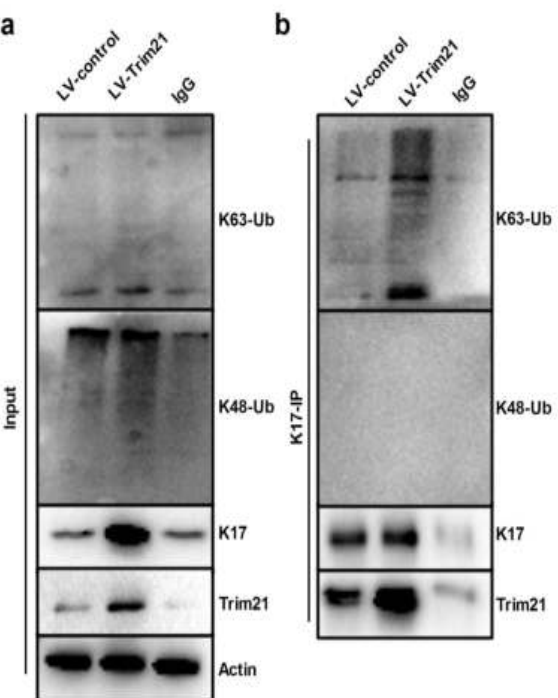

Fig.4. Trim21 ubiquitinates K17 via K63-linkage and promotes the proliferation of $\mathrm{HaCaT}$ cells. (a-b) Cells were transfected with lentiviral non-targeting control (LV-control) or LV-Trim21. Cells were subjected to immunoprecipitation with anti-K17 or IgG antibody and IB.

\section{CONCLUSION}

Taken together, our data suggest a previously unrecognized role
for ubiquitination in regulating protein level of K17 in psoriasis,
further indicating a novel mechanism in the pathogenesis of
psoriasis. Trim21, which regulated K17 activation by
ubiquitination, provides a potential target for interrupting K17
activation in the treatment of psoriasis.

ACKNOWLEDGEMENTS

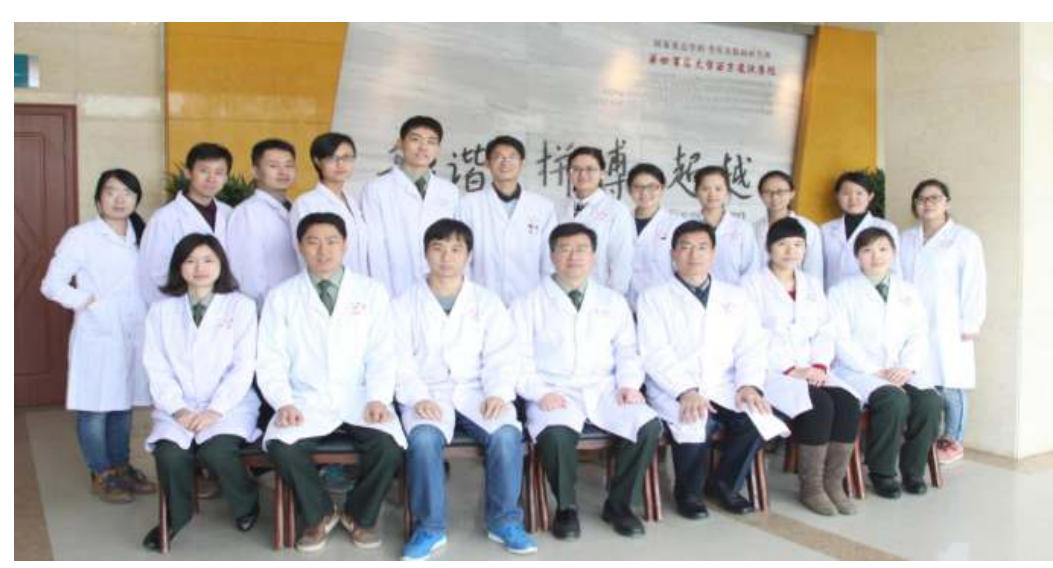

